\section{Optical monitoring of stem cell-substratum interactions}

\author{
Amirreza Aref, Robert Horvath, James McColl, ${ }^{*}$ and \\ Jeremy J. Ramsden \\ Cranfield University, Department of Materials, Bedfordshire \\ MK43 0AL, United Kingdom
}

\begin{abstract}
Modulation of the coupling of light into a waveguide via a grating, together with a novel approach to analyzing the data, is used to investigate the attachment of human embryonal carcinoma stem cells to three substrata: silica-titania (representative of artificial implants); polylysine (a commonly used laboratory cell culture substrate); and mucin (the coating of the mucosae). By considering both in-coupling peak width and position, the secretion of microexudate by the cells, the formation of filopodia, and the overall change in their shape (spreading) can be distinguished. This cannot be achieved by the conventional microscopic imaging approach. Moreover, we obtain the kinetics of these processes with excellent time resolution. (0) 2009 Society of Photo-Optical Instrumentation Engineers. [DOI: 10.1117/1.3065541]
\end{abstract}

Keywords: x-rays; microscopy; synchroton radiation; tissues; particles; fluorescence.

Paper 08267LRR received Aug. 4, 2008; revised manuscript received Nov. 18, 2008; accepted for publication Nov. 18, 2008; published online Feb. 11, 2009.

\section{Introduction}

The morphology of cell-substratum contacts is still poorly characterized, despite its importance (e.g., for cell development and biocompatibility ${ }^{1}$ ), because of the multiplicity of processes occurring simultaneously. The most obvious and characteristic change is that of overall cell shape, ${ }^{2}$ but there is good evidence for filopodia produced by the cell in the course of exploring and attaching itself to substrata, ${ }^{3}$ and for the production of extracellular secretions (microexudate) used by the cell to condition its immediate environment. ${ }^{4}$ These phenomena have been individually investigated in some detail, down to the molecular level, but only optical microscopy has yielded an overall view. Nevertheless, its relatively poor spatial and temporal resolution with respect to phenomena occurring at the nanoscale has limited full understanding of the overall process. Moreover, many of the microscopic techniques require the addition of fluorescent dye molecules ("labels") to the system in quantities sufficient to constitute invasion, with poorly defined effects on cell physiology, adding an extra layer of complication to any interpretation of the results.

A significant innovation was the exploitation of the evanescent field generated by total internal reflexion at the cell-

*Present address: University Chemical Laboratory, Lensfield Road, Cambridge, UK.

Address all correspondence to: Jeremy J. Ramsden, Cranfield University, Department of Materials, Bedfordshire MK43 OAL, UK. Tel. +44 1234 754100; Fax +44 1234 751346, E-mail: j.ramsden@cranfield.ac.uk. (and medium)-substratum interface to preferentially illuminate the region of cell-substratum contacts. ${ }^{5}$ In this technique, a fluorescent molecule is dissolved in the surrounding (aqueous) medium, ${ }^{6}$ and it is assumed that the label can freely diffuse into the contact region. Direct fluorescent labeling of the cell membrane has also been used to create a map of cell-substratum topography. ${ }^{7}$

More recently, imageless label-free techniques, exploiting the evanescent field created by light guided in optical waveguides, have been introduced, establishing that the size, shape, and average refractive index of the cell body can be determined. ${ }^{8,9}$ Since the evanescent field decays exponentially away from the surface of the waveguide (i.e., the substratum), what is actually sensed by the waveguide (via the propagation constants of its modes) is the Laplace transform of the cell cross section parallel to the substratum. ${ }^{10}$ It has been convincingly demonstrated that high resolution kinetics of the spreading transformation on different substrata can be obtained by this method. ${ }^{10}$

These nonimaging waveguide methods have now been augmented by a powerful new development that enables the arrangement of the cells on the substratum to be simultaneously characterized. ${ }^{11,12}$ In the most common arrangement, ${ }^{13}$ external light (a highly monochromatic, wavelength $\lambda$, linearly polarized laser beam several tenths of a millimeter in diameter) falls on a grating (1 to $2 \mathrm{~mm}$ long, grating constant $\Lambda$ ) incorporated in the waveguide for the purpose of coupling light into it. Sweeping the angle $\alpha$ between the incident light and the grating normal while measuring the intensity of the incoupled light using photodiodes pressed against the waveguide ends yields a series of peaks (called the mode spectrum) according to the in-coupling condition $N_{m}=\sin \alpha$ $+\lambda \ell / \Lambda$, where $N_{m}$ is the effective refractive index of a given lightmode $m=0,1, \ldots$, and $\ell=0, \pm 1,2, \ldots$ is the diffraction order. $N_{m}$ is a function of the refractive indices of the waveguide layers, ${ }^{13}$ including the cover material (comprising the cells and their surrounding liquid medium). The peaks have a finite width $w,{ }^{13}$ conveniently measured at half the maximum height. When cells are present on the grating, it is observed $^{11,12}$ that the broadening is much greater than the theoretically limiting peak width derived from the optical uncertainty principle. ${ }^{13}$ Depending on the actual construction of the waveguide, two peaks may even be observed. ${ }^{14}$ It is intuitively obvious that the greatest broadening is observed when exactly half the substratum is covered by cells, and this has been confirmed by detailed calculations, ${ }^{12}$ as well as experiments and simulations. ${ }^{11}$ For a given surface coverage $\theta$, the peak width also depends on the magnitude of the optical contrast between the covered and uncovered zones (i.e., the difference in refractive indices between the cells and the medium in which they are immersed). (The incoupling efficiency, and hence the peak heights, depends on the optical contrast between the waveguiding film and the cover material, which will also influence the composite peak width. ${ }^{11,12}$ )

The purpose of this work is to exploit these new theoretical developments. The waveguide surface, ultracleaned and in some cases coated, was seeded with a fixed number of spherical cells fresh from culture. We investigated three distinctively different cell substrata. Silica-titania is representative

1083-3668/2009/14(1)/010501/3/\$25.00 @ 2009 SPIE 


\section{JBO LETTERS}

of artificial implants (titanium is the most common material used for prostheses, ${ }^{1}$ and in the body is invariably oxidized; the salient surface energetic features, most notably the Lewis acid/base parameters, of titania are almost the same as those of silica). Polylysine is a very commonly used cell culture substrate in the laboratory. Mucin is found ubiquitously coating the mucosae (mucins comprise a family of heavily glycosylated, high molecular weight glycoproteins with carbohydrate side chains that make up some 50 to $80 \%$ of the molecule by weight). By simultaneously comparing the evolution of the in-coupling peak width with the evolution of the in-coupling peak position, we show that it is possible to separately determine microexudate secretion (which does not affect in-coupling peak width), filopodium formation, and somatic cell spreading.

\section{Experimental}

TERA2 human EC cell lines were cultured according to Ref. 15. Pharmaceutical-grade porcine gastric mucin (PGM, mean molecular weight $565 \mathrm{kDa}$ ) was purchased from A/S Orthana Kemisk Fabrik (Kastrup, Denmark). The commercial preparation was dialyzed to remove all salts and low molecular weight additives and lyophilized for storage. PLL (mean molecular weight $5 \mathrm{kDa}$ ) was used as received (Sigma).

Waveguides (see next paragraph) were ultracleaned at room temperature with chromic acid. The PLL $(0.01 \% \mathrm{w} / \mathrm{w})$ and mucin $(0.1 \% \mathrm{w} / \mathrm{w})$ solutions were made up by dissolving weighed dry material in ultrapure water and pre-equilibrated overnight. The solutions were applied to the ultracleaned waveguides for $20 \mathrm{~min}$ and then washed twice with ultrapure water [this procedure has been reproducibly found to completely coat the waveguides, ${ }^{14}$ and was verified directly using optical waveguide lightmode spectroscopy(OWLS)]. Coated and uncoated waveguides were incubated overnight in cell culture medium buffered with 10-mM HEPES-NaOH, $\mathrm{pH}$ 7.0. Cells were detached from the culture flask ${ }^{15}$ and carefully introduced into a specially designed open microcuvette $7 \mathrm{~mm}$ in diameter, of which the waveguide formed the bottom.

Waveguides $c a 180 \mathrm{~nm}$ thick were made from amorphous silica:titania at a ratio of approximately $2: 1$ (with a refractive index of approximately 1.75). They incorporated a shallow (5 to $10 \mathrm{~nm}$ ) grating coupler (type 2400, MicroVacuum, Budapest), grating constant equal to $416.667 \mathrm{~nm}$. The penetration depth of the evanescent field is of the order of $100 \mathrm{~nm}$. The in-coupling resonance peaks for the $\mathrm{TM}_{0}$ mode of the waveguides were measured every $40 \mathrm{~s}$ using a laboratory-built setup with a resolution of better than $10^{-5}$ radians ${ }^{16,17}$ and were saved for subsequent analysis. The peak position and overall width were defined and determined according to Ref. 12.

\section{Results and Discussion}

Figure 1 shows the microscope images of the cells on the three different substrata. Even visually, it is apparent that the cells have not spread on the mucin, have significantly spread in a circularly symmetrical fashion on the PLL, and have spread in an elongated fashion on the $\mathrm{Si}(\mathrm{Ti}) \mathrm{O}_{2}$.

The corresponding kinetic data showing the evolution of the in-coupling angle $\alpha$ (directly related to $N$ ) and the incoupling peak width $w$ are shown in Fig. 2 .
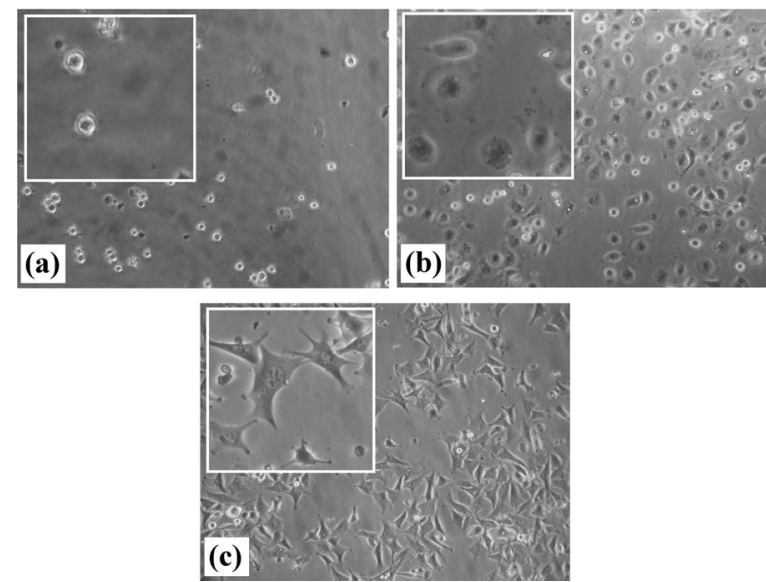

Fig. 1 Optical micrographs of TERA2.spl2 stem cells on (a) mucin, (b) $\mathrm{PLL}$, and (c) $\mathrm{Si}(\mathrm{Ti}) \mathrm{O}_{2}$ after $120 \mathrm{~min}$ of culture. Each main image is $850 \mu \mathrm{m}$ wide, and the insets are $170 \mu \mathrm{m}$ wide.

Figure 2(a) confirms and quantifies the visual observations (Fig. 1). Figure 2(b) provides much stronger evidence for the absence of spreading on mucin, since there is no change in in-coupling peak width. Furthermore, in Fig. 2(a) the kinetics of increase of propagation constant are definitely sigmoidal (except with mucin), the shape that appears invariably to be associated with cell spreading (see Ref. 10). The most plausible explanation of the small and slow hyperbolic increase in $\Delta \alpha$ for the cells on mucin is that it is due to microexudate (i.e., the extracellular matrix molecules secreted by the cell in
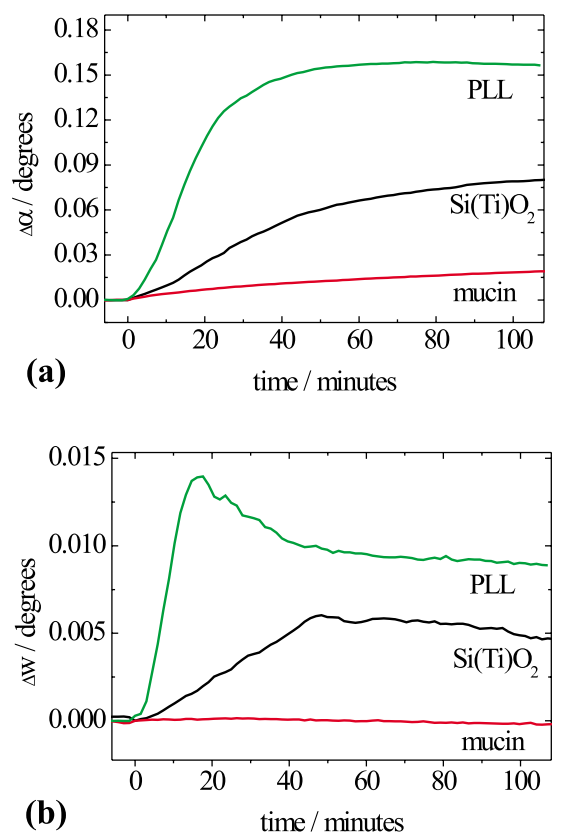

Fig. 2 (a) In-coupling angle, given as the difference $\Delta \alpha$ between the actual value and the value immediately prior to seeding the waveguide with $5 \times 10^{4}$ TERA2.spl 2 stem cells, grown for at least two days with one passage, in a total volume of $550 \mu \mathrm{L}$ on (from top to bottom) PLL, $\mathrm{Si}(\mathrm{Ti}) \mathrm{O}_{2}$, and mucin. (b) In-coupling peak width, given as the difference $\Delta w$ between the actual value and the value immediately prior to seeding the waveguide with the cells. 


\section{JBO LETTERS}

an attempt to render an uncongenial substratum congenial). Protein deposition kinetics usually give such hyperbolic kinetics (see Ref. 18).

The evolution of $\Delta w$ is predicted to reach a maximum when $50 \%$ of the waveguide surface is covered by objects. ${ }^{14}$ That cells reach this maximum much faster on PLL than on $\mathrm{Si}(\mathrm{Ti}) \mathrm{O}_{2}$ is not surprising, given the more rapid evolution of $\Delta \alpha$. What is revealing is that the value of $\Delta \alpha$ at the moment when $\Delta w$ reaches its maximum is much greater for PLL than for $\mathrm{Si}(\mathrm{Ti}) \mathrm{O}_{2}$. We propose that this indicates that the cellsubstratum contact is stronger (especially through more filopodia) for the former than for the latter. The fact that the height of the $\Delta w$ maximum is much greater for the PLL corroborates this inference, for it implies that the optical contrast between the cell-covered and uncovered regions of the waveguide is greater.

Comparison of Fig. 3 with Fig. 2 strikingly shows the effect of just one passage on cell behavior. First, for the initial 20 min the temporal evolution of $\Delta \alpha$ for the cell not having undergone passage is hyperbolic, not sigmoidal, i.e., characteristic of protein deposition, but is sigmoidal thereafter. Second, there is a $30-\mathrm{min}$ lag before $\Delta w$ starts to increase; protein secretion, being uniform, cannot cause $w$ to increase. We estimate the amount of protein after $30 \mathrm{~min}$ to be roughly $1.6 \mathrm{ng} / \mathrm{mm}^{2}$ (using $\partial M / \partial \Delta \alpha$ from Ref. 18). From the rather low value of the $\Delta w$ peak height, we also infer that the cellsubstratum contact is weaker (hence giving a lower optical contrast) than for cells that have undergone one passage. This can be made quantitative: we propose that the value of $\Delta \alpha$ at the instant when $\Delta w$ is at its peak is equated with the cellsubstratum adhesivity (a composite quantity indicating the strength of the cell-substratum contact).

In conclusion, simultaneous examination of both the incoupling peak position and width arising from cells on a waveguide allows the contributions of the shape (and size and composition, but in the present experiments these did not change significantly during our measurements) of the cells to the perturbation of the evanescent field of the waveguide to be separated from that of macromolecules secreted by the cells and deposited on the waveguide surface. The methodology described in this work can be anticipated to be of value for high-throughput experiments (see Ref. 19), since a single set of simultaneous measurements, not requiring labeling, yields the rate of cell spreading, the rate of macromolecular secretion, and the adhesivity (related to the density of filopodia).

\section{References}

1. J. J. Ramsden, Biomedical Surfaces, Artech House, Norwood MA (2008).

2. A. S. G. Curtis, "The mechanism of adhesion of cells to glass," $J$. Cell Biol. 20, 199-215 (1964).

3. K. Burridge, K. Fath, T. Kelly, G. Nuckolls, and C. Turner, "Focal adhesions," Annu. Rev. Cell Biol. 4, 487-525 (1988).

4. G. Poste, L. W. Greenham, L. Mallucci, P. Reeve, and D. J. Alexander, "The study of cellular microexudates by ellipsometry and their relationship to the cell coat," Exp. Cell Res. 78, 303-313 (1973).

5. D. Axelrod, "Cell-substrate contacts illuminated by total internal re-

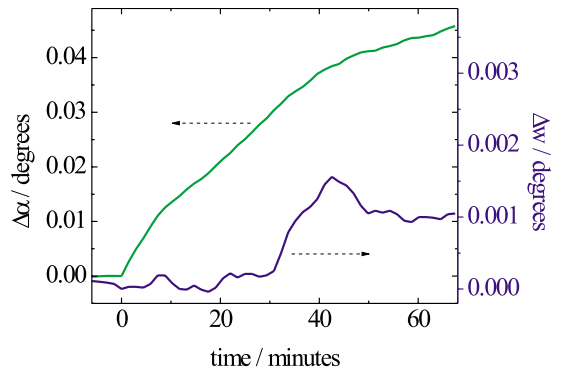

Fig. 3 Plot of in-coupling angle (left-hand scale) and the in-coupling peak width (right-hand scale) following the seeding of a PLL-coated waveguide (at $t=0$ ) with $5 \times 10^{4}$ TERA2.spl2 stem cells not having undergone passage.

flection fluorescence," J. Cell Biol. 89, 141-145 (1981).

6. D. Gingell, O. S. Heavens, and J. S. Mellor, "General electromagnetic theory of total internal reflection fluorescence: the quantitative basis for mapping cell-substratum topography," J. Cell. Sci. 87, 677693 (1987).

7. J. S. Burmeister, G. A. Truskey, and W. M. Reichert, "Quantitative analysis of variable-angle total internal reflection fluorescence microscopy (VA-TIRFM) of cell/substrate contacts," J. Microsc. 173, 39-51 (1994).

8. S. Y. Li, J. J. Ramsden, J. E. Prenosil, and E. Heinzle, "Measurement of adhesion and spreading kinetics of baby hamster kidney and hybridoma cells using an integrated optical method," Biotechnol. Prog. 10, 520-524 (1994).

9. T. S. Hug, J. E. Prenosil, and M. Morbidelli, "Optical waveguide lightmode spectroscopy as a new method to study adhesion of anchorage-dependent cells as an indicator of metabolic state," Biosens. Bioelectron. 16, 865-874 (2000).

10. J. J. Ramsden, S. Y. Li, E. Heinzle, and J. E. Prenosil, "An optical method for the measurement of number and shape of attached cells in real time," Cytometry 19, 97-102 (1995).

11. R. Horvath, J. Vörös, R. Graf, G. Fricsovszky, M. Textor, L. R. Lindvold, N. D. Spencer, and E. Papp, "Effect of patterns and inhomogeneities on the surface of waveguides used for optical waveguide lightmode spectroscopy applications," Appl. Phys. B 72, 441-447 (2001).

12. K. Cottier and R. Horvath, "Imageless microscopy of surface patterns using optical waveguides," Appl. Phys. B 91, 319-327 (2008).

13. K. Tiefenthaler and W. Lukosz, "Sensitivity of grating couplers as integrated-optical chemical sensors," J. Opt. Soc. Am. B 6, 209-220 (1989).

14. R. Horvath, H. C. Pedersen, N. Skivesen, D. Selmeczi, and N. B. Larsen, "Monitoring of living cell attachment and spreading using reverse symmetry waveguide sensing," Appl. Phys. Lett. 86, 071101 (2005).

15. S. A. Przyborski, "Derivation and culture of human embryonal carcinoma stem cell line," in Culture of Human Stem Cells, R. I. Freshney, G. N. Stacey, and J. M. Auerbach, Eds., p. 133, Wiley, Chichester, UK (2007).

16. R. Horvath, G. Fricsovszky, and E. Papp, "Application of optical waveguide lightmode spectroscopy to monitor lipid bilayer phase transitions," Biosens. Bioelectron. 18, 415-428 (2003).

17. R. Horvath, H. C. Pederson, and N. B. Larsen, "Demonstration of reverse symmetry waveguides sensing in aqueous solutions," Appl. Phys. Lett. 81, 2166-2168 (2002).

18. J. J. Ramsden, "Concentration scaling of protein deposition kinetics," Phys. Rev. Lett. 71, 295-298 (1993).

19. Y. Fang, A. M. Ferrie, N. H. Fontaine, J. Mauro, and J. Balakrishnan, "Resonant waveguide grating biosensor for living cell sensing," Biophys. J. 91, 1925-1940 (2006) 